\section{A more flexible mouse}

To understand how neuronal circuits work, we also need to know about their anatomy. The discovery, around 20 years ago, that the plant lectin wheatgerm agglutinin (WGA) can be transported anterogradely along axons and across synapses, led to the development of transneuronal tracing techniques to visualize neuronal pathways and circuits. More recently, transgenic mice have been generated that express WGA under the control of tissue-specific gene promoters, allowing circuits to be traced from defined regions of the central nervous system (CNS). However, making a different transgenic line for each experiment is very laborious, and the range of good regionspecific promoters is limited. To address these problems, Braz et al. have engineered a transgenic mouse that allows a more flexible approach.

The authors constructed a gene-expression vector in which the $W G A$ gene was positioned downstream of a $L a c Z$ reporter gene. The expression of $L a c Z$ was driven by the chicken $\beta$-actin promoter, which is active in most tissues. The $L a c Z$ coding sequence was flanked with loxP sites, so that it could be excised in the presence of Cre recombinase, thereby bringing the $W G A$ gene under the control of the $\beta$-actin promoter. The authors used this vector to create a transgenic mouse line, which they named ZW. By breeding these mice with lines in which different promoters controlled Cre recombinase expression, they could express WGA in a tissue-specific manner. As examples, they traced the neuronal pathways from the eye and the cerebellum by expressing Cre from retinal and Purkinje-cell-specific promoters, respectively.

Of course, the applications of this technique will be limited by the availability of mouse lines that express Cre in an appropriate pattern. However, the ZW mouse has another advantage; the WGA gene can also be activated at any time and in any region of the CNS, by injection of a Cre-expressing viral vector. To demonstrate the efficacy of this technique, the authors used it to trace neuronal circuits in the cerebellum and visual cortex of adult mice.

Although the $\beta$-actin promoter is generally thought to be ubiquitously active, Braz et al. found that it caused WGA to be expressed in a mosaic pattern in the ZW mice. However, they managed to turn this potential drawback to their advantage, by showing that it enabled individual neurons to be distinguished from their unlabelled neighbours. This provided a single-cell resolution that was comparable to Golgi staining, with the bonus of making it possible to study connectivity as well as morphology.

As transgenic mouse lines that express Cre recombinase from tissue-specific promoters become more widely available, it will be possible to use the $\mathrm{ZW}$ mouse to trace neuronal circuits in a wide variety of brain regions. The fact that the activation of the WGA gene can also be controlled in space and time using a
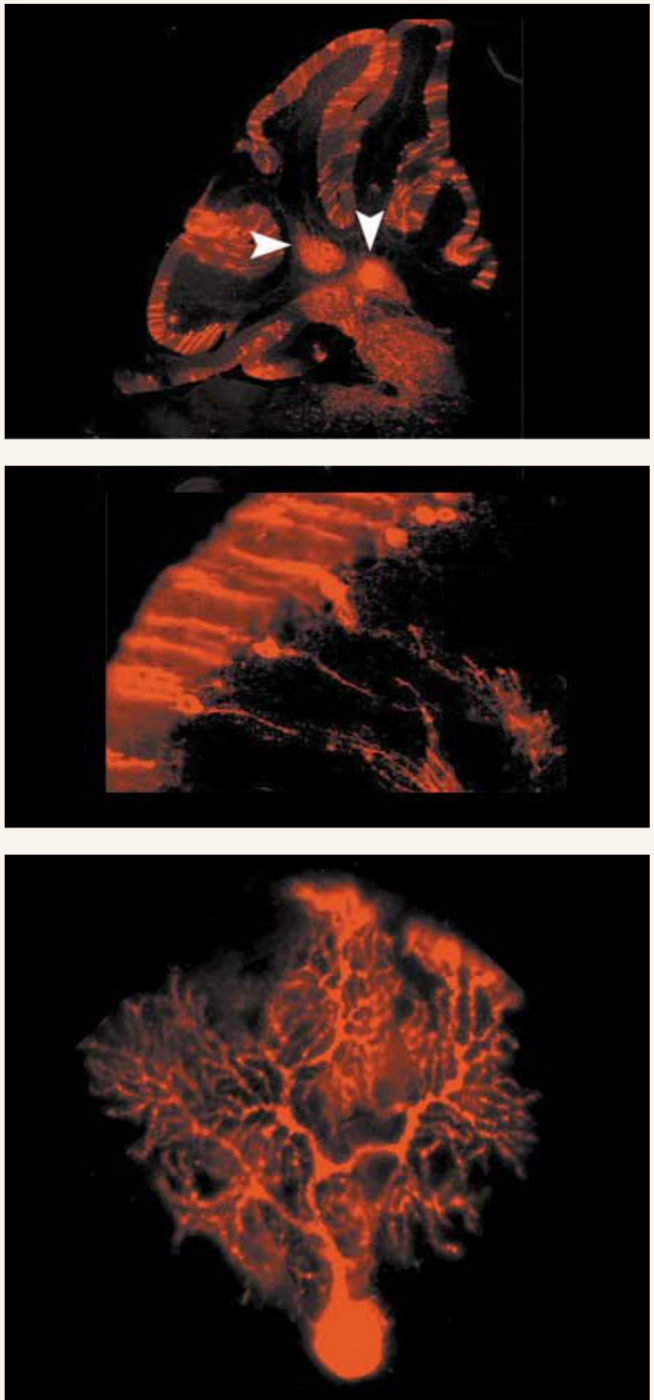

WGA expression in the cerebellum of ZW transgenic mice. The WGA immunostaining is found in Purkinje cells (top and middle panels), and axonal branches are detected in the deep cerebellar and vestibular nuclei (arrowheads). () 2002 National Academy of Sciences, USA. The bottom panel shows the morphology of a WGA-filled Purkinje cell, visualized by immunostaining. Courtesy of Allan Basbaum, University of California, San Francisco, California, USA.

Cre-expressing viral vector provides an additional level of flexibility, making this mouse an extremely valuable tool for visualizing both developing and adult neuronal circuits.

Heather Wood

6.) References and links

ORIGINAL RESEARCH PAPER Braz, J. M. et al. Transneuronal tracing of diverse CNS circuits by Cre-mediated induction of wheat germ agglutinin in transgenic mice. Proc. Natl Acad. Sci. 99, 15148-15153 (2002) FURTHER READING Ruda, M. \& Coulter, J. D. Axonal and transneuronal transport of wheat germ agglutinin demonstrated by

immunocytochemistry. Brain Res. 249, 237-246 (1982) | Lewandoski, M. Conditional control of gene expression in the mouse. Nature Rev. Genet. 2 743-755 (2001)
Prions, prions everywhere Once again, prion diseases are in the news. In the UK, as we go to press, the High Court is about to rule on whether two patients can be given an experimental treatment for CreutzfeldtJakob disease (CJD). According to $B B C$ News Online (6 December), both of the patients - a 15-year-old girl and an 18-year-old man - are dying of variant CJD. Their parents are fighting for the right to have them treated by intracerebral injections of pentosal polysulphate, which studies suggest might be able to interact with prions and prevent further damage. Although the parents have found doctors who are willing to give the treatment, the administration of such an 'unknown quantity' to patients who cannot give informed consent is a difficult issue. If the treatment goes ahead, the results will be watched with keen interest by both clinicians and patients' families.

Meanwhile, in the United States, the Dining and Wine section of the New York

Times asks the question, 'Is American venison safe?' (4 December). As chronic wasting disease (CWD), a prion disorder that affects deer and elk, spreads now affecting 11 states and 2 Canadian provinces there is concern over whether it could cross the species barrier to humans, in the same way as bovine spongiform encephalopathy is thought to have caused variant CJD in Europe.

Although there is no evidence that eating infected venison can cause disease in humans, the publication Consumer Reports on Health (December) has stated that "eating deer and elk may be risky". Many restaurants and suppliers have switched to venison from New Zealand, where CWD has not been found, and it is likely that many consumers will play it safe and avoid venison altogether. 